\title{
Face Recognition Using Support Vector Model Classifier for User Authentication
}

\author{
Wen-Hui Lin ${ }^{1 *}$, Ping Wang ${ }^{1}$, Chen-Fang Tsai ${ }^{2}$ \\ ${ }^{1}$ Department of Information Management \\ Kun Shan University, Tainan, Taiwan \\ linwh@mail.ksu.edu.tw, \\ pingwang@mail.ksu.edu.tw \\ ${ }^{2}$ Department of Industrial Engineering Management, \\ Aletheia University, Tanshui,, Taiwan \\ au1204@mail.au.edu.tw
}

\begin{abstract}
Most existing user authentication approaches for detecting fraud in e-commerce applications have focused on Secure Sockets Layer (SSL)-based authentication to inspect a username and a password from a server, rather than the inspection of personal biometric information. Because of the lack of support for mutual authentication or twoway authentication between a consumer and a mercantile agent, one-way SSL authentication cannot prevent man-in-the-middle attacks. In practice, in user authentication systems, machine learning and the generalisation capability of support vector models (SVMs) are used to guarantee a small classification error. This study developed an online face-recognition system by training an SVM classifier based on user facial features associated with wavelet transforms and a spatially enhanced local binary pattern. A cross-validation scheme and SVMs associated with the Olivetti Research Laboratory database of user facial features were used for solving classification precision problems. Experimental results showed that the classification error decreased with an increase in the size of the training samples. By using the aggregation of both the low-resolution and the high-resolution face image samples, the global precision of face recognition was over $97 \%$ with tenfold cross-validation scheme for an image data size of 168 and 341, respectively. Overall, the proposed scheme provided a higher precision of face recognition compared with the average precision for low-resolution face image (approximately 89\%) of the existing schemes.
\end{abstract}

Keywords: e-commerce; SVM; face recognition; wavelet transforms; local binary pattern

\section{Introduction}

E-commerce applications have been increasing rapidly. Service providers are expected to guarantee secure transaction authentication for Web services. While Web services provide e-commerce applications that offer valuable opportunities, Web services face major challenges since consumers are reluctant to avail e-commerce services in the absence of service provider guarantees regarding the security of their information. In particular, Secure Sockets Layer (SSL)-based authentication provides an efficient and economical means of using the standard username/password convention. However, one-way SSL authentication cannot prevent cyber attacks 
such as phishing and man-in-the-middle attacks, which is a major obstacle to consumer adoption of e-commerce. Generally, when using a banking mobile app to access banking services online, people never think that they could become a victim of identity theft. According to reports released by privacy IT Security in the United States, hackers had gained root access to 90 servers of a financial institution's servers in 2014, and the access enabled the hackers to transfer funds, disclose customer information, open new accounts, and even close accounts without prior knowledge of the affected customers (IT Security 2015). Because of the nondenial requirements of remote user identity authentication schemes, such access is most commonly achieved using a biometricsbased approach.

Identity theft is therefore one of the most severe threats to the security of online transactions associated with e-commerce services. Consequently, it is imperative that service providers have the means to authenticate the identity of every user for detecting fraud. Efficient user authentication schemes are required to build trust in e-commerce, and mutual authentication schemes are crucial for e-commerce applications. As the sophistication of tools used by malicious users continues to increase, the data processed in e-commerce are at increased risk of attack. Consequently, there is an urgent need for robust authentication schemes that can confine data access to legitimate, authorised users by preventing malware from tracking transactions during authenticated sessions.

Multifactor authentication approaches in which digital certificates are provided to users by a public key infrastructure mechanism have been developed for user authentication systems. These approaches enable detecting fraudulent use of user identity because they involve using face-recognition information, radio frequency identification (RFID) tags, and machine learning (ML) techniques. Numerous twofactor improvement methods (Min et al. 2011, Jing et al. 2009, Nguyen et al. 2012, Yang et al. 2012, Battaglia et al. 2014) have been proposed. Authors incorporated a face-recognition feature into a smart card, and images of each user with different facial expressions were stored in a server database and used as the basis for identity authentication. The front-end smart cards served as a secondary source and stored only a small amount of the identity information.

The online face-recognition system proposed in this study employs a support vector model (SVM) classifier and has the following advantages: (i) It provides privacy protection by classifying users by using a signature-based SVM classifier (SVC) based on multilevel wavelet transformation; multilevel wavelet transformation, which is also used in the face image approach, involves using a spatially enhanced local binary pattern (LBP) (Mirza et al. 2013) of a user feature and enables accurately determining the user identity in online transactions. (ii) The proposed scheme uses a complete face image rather than partial image information to increase the recognition precision. (iii) The multilevel wavelet transformation of the face images enables the system to perform hierarchical decision-making, which increases the flexibility of the system. Experimental results reveal that the proposed system provides a secure approach for protecting a user's biometric privacy and achieves high-precision face recognition, features that are crucial in e-commerce security mechanisms.

The remainder of this paper is organised as follows: Section 2 reviews previous studies in the fields of face recognition and ML. Section 3 introduces the model that was used to construct the proposed recognition system. In Section 4, the proposed 
approach is presented by considering the case of a user with various facial expressions, and the approach is demonstrated by employing it in an e-commerce security system. Section 5 examines the Radial Basis Function (RBF) kernel function for using the SVM approach in the proposed approach. Finally, Section 6 provides concluding remarks.

\section{Relate Work}

This section reviews the use of three crucial techniques-SVMs, face-recognition schemes for user authentication, and wavelet transforms - applied in face recognition for biometric authentication.

\subsection{Support Vector Models}

SVMs are used for clustering data into two categories according to maximum boundary geometry. SVMs are supervised learning models associated with learning algorithms, and they are used to analyse data and recognise patterns. Generally, the results from SVM classification algorithms are more accurate than those derived from other ML approaches involving nonoptimised search methods, such as those involving artificial neural networks, least squares, k-nearest neighbour, Bayesian probability, and classification and regression trees, particularly when collect only limited training data. In an SVM training algorithm, examples are assigned to a category depending on whether nonlinear or linear binary classifiers are obtained from a set of training examples. SVMs have been shown to be useful tools for performing clustering and classification analyses (An and Liang 2013, Abe S. 2015). In particular, SVM theory has been developed gradually from linear SVCs to hyperplane classifiers (Devi et al. 2015); in other words, SVMs can efficiently perform nonlinear classification by using a kernel function, and their inputs can be mapped into high-dimensional feature spaces by selecting an appropriate kernel function. Furthermore, a favourable classification result is achieved by using a hyperplane that is the farthest from the nearest training data point of any class (Wikipedia 2015). The basic SVM theory is as follows. (Vapnik 1995)

Consider a given training dataset $D\left(x_{i}, y_{i}\right)$, where $x_{i}$ denotes $n$ observations of malware signatures $\left(x_{i} \in R^{N}, i=1, \ldots, N\right)$ and $y_{i}$ is the corresponding class label of which the value is either 1 or -1 (i.e., malicious or benign); in other words, $y_{i}$ indicates the class to which the point $x_{i}$ belongs, with $y_{i} \in\{1,-1\}$, and a $y_{i}$ is assigned to each observation $x_{i}$. Each facial feature $x_{i}$ is of dimension $d$, which corresponds to the number of propositional variables.

$$
D=\left\{\left(x_{i,} y_{i}\right) \mid x_{i,} \in R^{N}, y_{i} \in\{1,-1\}\right\}_{i=1}^{N} \text {. }
$$

A typical clustering problem is the determination of the maximum margin of a hyperplane that divides the points corresponding to $y_{i}=1$ from those corresponding to $y_{i}=-1$. Any hyperplane can be written as the set of points $x$ satisfying the following formula: 


$$
w \cdot x_{i}+b=0 \quad \forall i
$$

where the dot in the first term denotes the dot product and $W$ denotes the normal vector of the hyperplane. The parameter $\frac{b}{\|w\|}$ determines the offset of the hyperplane from the origin in the direction of the normal vector $W$. Generally, a decision function $D\left(x_{i}\right)$ is defined for clustering, with $D\left(x_{i}\right)=w \cdot x_{i}+b$.

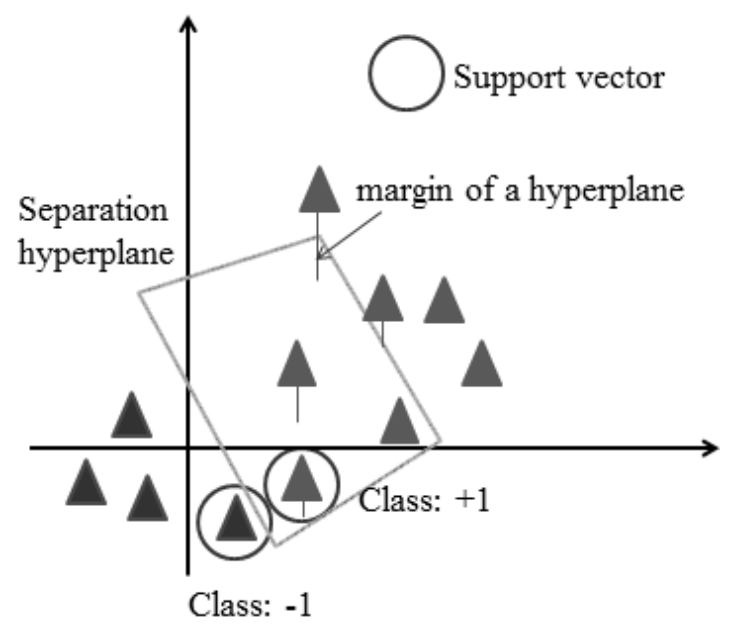

Figure 1. Determination of the maximum margin of a hyperplane(Wikipedia 2015).

As shown in Fig. 1, the Lagrange multiplier in the dual optimisation theory was used to determine the maximal and minimal optimisation functions, which provided a viable solution. To solve the problem of identifying the maximum margin of a hyperplane, the Lagrange function is expressed as follows:

$$
L_{P}=L(w, l)=\frac{\|w\|^{2}}{2}-\sum_{i=1}^{N} l_{i}\left[y_{i}\left(w \cdot x_{i}\right)+b\right]-\sum_{i=1}^{N} l_{i}
$$

where $l_{i}$ represents the Lagrange parameter. Theoretically, solving the problem of maximising the geometric boundary requires seeking the minimum of the normal $\left|w^{2}\right|$, which can be transformed to minimise the Lagrange optimisation function $L_{\mathrm{p}}$ subject to the constraint $y_{i}\left(w \cdot x_{i}\right)+b-1 \geq 0$ :

$$
\begin{gathered}
M I N L_{P} \quad \forall i \\
\text { subject to } y_{i}\left(w \cdot x_{i}\right)+b-1 \geq 0 \quad \forall i, \\
\text { with } l_{i} \geq 0 \quad \forall i .
\end{gathered}
$$

To obtain the feasible solutions by using the Lagrange dual optimisation theory, a set of support vectors $x_{i}$ satisfying two constraints according to the classification 
decision function $D(x): \operatorname{sgn}\left[\sum_{i=1}^{N} l_{i}\left(y_{i}\left(w x_{i}\right)+b\right)\right]$ must be identified.

A traditional linear SVM has a major drawback: assuming that the training data are linearly separable, the SVM cannot be valid when applied to practical real cases. Bernhard et al. (1992) suggested a novel approach to generating nonlinear classifiers; in the approach, a kernel function is used to obtain the maximum margins of hyperplanes. The nonlinear classification algorithm is formally similar to the linear SVM, except that each dot product (i.e., $\left.\phi\left(x_{i}\right) \bullet \phi\left(x_{j}\right)\right)$ is replaced by a kernel function.

This enables the algorithm to map the maximum margin hyperplane in a transformed feature space (i.e., $\Phi: R_{d} \rightarrow F$ ). However, rationally selecting a suitable mapping function $\Phi$ is a research topic that must be discussed.

The effectiveness of the SVM depends on the kernel selected and the parameters of the kernel. The considerations for selecting kernel functions are listed in Table 1. As shown in Table 1, the linear (Gunn 1998) and the radial basis function(RBF) (Buhmann 2003) were used as the kernel function of the SVC to establish the facerecognition system after the features and limitations of the four kernel functions were considered. To improve classification accuracy, a combination of two model parameters (i.e., soft margin parameter $C$ and gamma $\gamma$ ) for the RBF was regularly selected using a grid search scheme to determine the optimised parameters of an SVC. The final model, which was used for testing and classifying new data, was then trained on the entire training set by using the kernel parameters.

Table 1. Considerations for selecting the kernel function in LIBSVM (Hsu 2002)

\begin{tabular}{|l|l|l|}
\hline Kernel & \multicolumn{1}{|c|}{ Kernel function } & \multicolumn{1}{c|}{ Features } \\
\hline $\begin{array}{l}\text { Linear, } \\
\text { 1998) }\end{array}$ & $u^{\prime} v$ & $\begin{array}{l}\text {.A special case of RBF. } \\
\text { - Solving the optimisation problem for a linear } \\
\text { kernel is much faster. It can be applied when the } \\
\text { real-time requirement for fast facial recognition } \\
\text { is considered. }\end{array}$ \\
\hline $\begin{array}{l}\text { Polynomial, } \\
\text { (Goldberg } \\
\text { and } \\
\text { Elhadad } \\
\text { 2008) }\end{array}$ & $\left(r * u^{\prime} v+\text { coef } 0\right)^{\text {degree }}$ & $\begin{array}{l}\text {.Although the polynomial kernel requires more } \\
\text { model parameters than the kernel of RBF, it } \\
\text { might diverge in the convergence phase of the } \\
\text { resolution process. }\end{array}$ \\
\hline $\begin{array}{l}\text { Radial } \\
\text { Basis } \\
\text { Function, } \\
\text { (Buhmann, } \\
\text { 2003) }\end{array}$ & $\exp \left(-r^{*}\left|u^{\prime}-v\right|^{2}\right)$ & $\begin{array}{l}\text {.Only two model parameters, C and } \gamma, \text { are } \\
\text { required in the clustering process. } \\
\text {.RBF has been employed in a wide variety of } \\
\text { applications. }\end{array}$ \\
\hline $\begin{array}{l}\text { Sigmoid } \\
\text { (Lin and } \\
\text { Lin 2003) }\end{array}$ & $\tanh \left(r * u^{\prime} v+\right.$ coef 0$)$ & $\begin{array}{l}\text {.Suitable for classifying nonlinear data } \\
\text {.This approach cannot give a grantee on the } \\
\text { convergence of solution in the resolution } \\
\text { process. }\end{array}$ \\
\hline
\end{tabular}

2.2 Face-recognition Schemes for User Authentication in Electronic Commerce

Automated biometric-recognition systems, which are based on the unique physiological features of users, are now widely used in the automotive, information 
technology, and banking industries. In general, the physiological characteristics include the individual's shape of face fingerprint, vein pattern, and DNA.

Certain fraud methods involve acquiring a users' private information by using copied or hedge techniques, such as smart card duplication and identity theft. Two fundamental issues arise when users applying face-recognition approach to ecommerce. First, if client data is to be processed thru the Internet, it must be encrypted to ensure its privacy. As a result, efficient key management schemes are required to enable the encryption on users' private information. Second, as the sophistication of the tools used by malicious users continues to increase, the data processed is at increasing risk of attack. Consequently, there is an urgent requirement for robust remote authentication schemes including biometric authentication system based on individual's physiological characteristics to ensure that the data can be accessed only by legitimate, authorized users.

Automatically detecting malicious users for preventing unauthorised access is a challenging task for an online e-commerce security system. Many face-recognition approaches involve LBP algorithms for user authentication. Numerous facerecognition techniques have been used in analysis algorithms for achieving high authentication precision. They include principal component analysis (Ahonen et al. 2006) and approaches involving LBPs (Jing et al. 2009), local ternary patterns (LTPs) (Tan and Triggs 2010), and spatially enhanced LBPs (Mirza et al. 2013). These facerecognition schemes are summarised in Table 2.

Table 2. Face-recognition approaches for user authentication

\begin{tabular}{|c|c|c|}
\hline Author & Features & Suitable for \\
\hline $\begin{array}{l}\text { Ojala et al. } \\
(1996)\end{array}$ & $\begin{array}{l}\text { Proposed a highly effective method } \\
\text { that employed LBPs for a regional } \\
\text { description of texture. }\end{array}$ & $\begin{array}{l}\text { Ojala et al. evaluated the } \\
\text { performance of texture measures } \\
\text { that have been successfully used } \\
\text { in various applications, and of } \\
\text { some new promising } \\
\text { approaches. }\end{array}$ \\
\hline $\begin{array}{l}\text { Ahonen et } \\
\text { al.(2006) }\end{array}$ & $\begin{array}{l}\text { Presented a novel and efficient } \\
\text { facial image representation based } \\
\text { on LBP texture features. }\end{array}$ & $\begin{array}{l}\text { The face image is divided into } \\
\text { several regions from which LBP } \\
\text { feature distributions are } \\
\text { extracted and concatenated into } \\
\text { an enhanced feature vector that } \\
\text { is used as a face descriptor. }\end{array}$ \\
\hline Liao (2010) & $\begin{array}{l}\text { Enhanced local texture feature sets } \\
\text { for face recognition by using the } \\
\text { strong antinoise capability of LTPs. } \\
\text { The face image is vulnerable to } \\
\text { noise and changes in illumination. }\end{array}$ & $\begin{array}{l}\text { Liao proved that centre- } \\
\text { symmetric extended local } \\
\text { ternary patterns (CS-LTPs) have } \\
\text { recognition capability, antinoise } \\
\text { capability, and processing speed } \\
\text { to meet engineering needs. }\end{array}$ \\
\hline $\begin{array}{l}\text { Min et al. } \\
(2011)\end{array}$ & $\begin{array}{l}\text { Developed a system that can } \\
\text { improve the safety of the RFID } \\
\text { system. The system involves two- } \\
\text { step authentication procedures for } \\
\text { enhancing the security of general } \\
\text { RFID systems. }\end{array}$ & $\begin{array}{l}\text { The system improves the } \\
\text { security of an automatic entrance } \\
\text { and exit authentication system; } \\
\text { an RFID tag and features } \\
\text { extracted from a user image are } \\
\text { used in the system. }\end{array}$ \\
\hline
\end{tabular}




\begin{tabular}{|l|l|l|}
\hline $\begin{array}{l}\text { Yang et al. } \\
(2012)\end{array}$ & $\begin{array}{l}\text { Proposed a new privacy-preserving } \\
\text { authentication method involving } \\
\text { encrypted biometric information } \\
\text { such as a fingerprint or an iris } \\
\text { signature for comparison. }\end{array}$ & $\begin{array}{l}\text { This scheme has advantages } \\
\text { such as high security, low } \\
\text { computation complexity, and } \\
\text { privacy of the biometric } \\
\text { template. }\end{array}$ \\
\hline & $\begin{array}{l}\text { Proposed VisilabFaceRec, a } \\
\text { multifactor authentication system } \\
\text { based on a combination of a dual- } \\
\text { Battaglia et } \\
\text { al.(2014) }\end{array}$ & $\begin{array}{l}\text { VisilabFaceRec does not require } \\
\text { using a centralised database and } \\
\text { stores sensitive data in the RFID } \\
\text { biometric identification (face } \\
\text { recognition) and an encrypted } \\
\text { RFID tag for token-based } \\
\text { authentication. }\end{array}$ \\
\hline
\end{tabular}

As shown in Table 2, most existing proposals are invariably based on the assumption of employee honesty. In practice, this assumption cannot also be guaranteed in practical applications, and many real cases have been reported in which fraudulent interior staffs have stolen clients' authentication details including biometric information from the signature server for financial gain. To resolve the security issues, the present study proposes a new remote authentication scheme based on a secretsplitting concept (Wang et al. 2011) with distributed facial features for an online ecommerce security system.

\subsection{Wavelet Transforms}

The fundamental concept of wavelet transform is that the transform should permit changes only in time extension, but not in the shape, on the basis of suitable basis functions. In mathematics, a wavelet series is the representation of a real- or complexvalued function by an orthonormal series that is generated by a wavelet (Charles 1992). Currently, wavelet transformation is one of the most prevalent time-frequency transformation techniques. This paper provides a formal, mathematical definition of an orthonormal wavelet and of the integral wavelet transform. In practice, a wavelet transform is a multiresolution wavelet-analysis technique. During first-order decomposition, the face image is subjected to an $\mathrm{LL}_{1}, \mathrm{LH}_{1}, \mathrm{HL}_{1}$, or $\mathrm{HH}_{1}$ band of decomposition, as shown in Fig. 2. Fig. 2(a) shows the first and second orders of decomposition based on wavelet transformation; each cell has a different resolution. Fig. 2(b) shows examples of the decomposition shown in Fig. 2(a), and $\mathrm{LL}_{2}$ represents a decomposed second-order image in Fig. 2(b-1). Wavelet conversion decomposes a face image into two parts for feature extraction: (i) a lower-resolution image and (ii) a higher-resolution image. The more common facial features appear as part of the lowerresolution information, whereas the high-resolution portion contains most of the facial features, such as local variations in illumination, expression, and dress. Conversely, inverse wavelet transformation combines the two parts of the face image into the original image through an information-reconstruction process. 


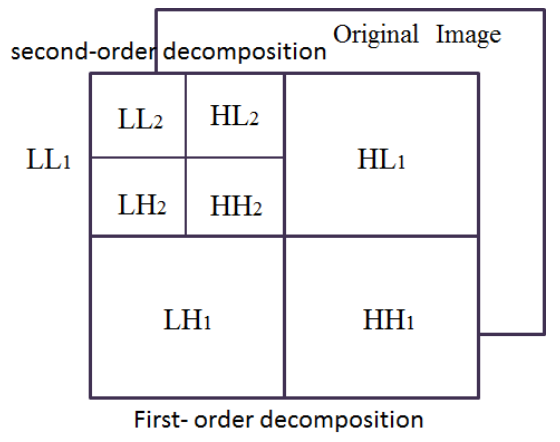

(a)

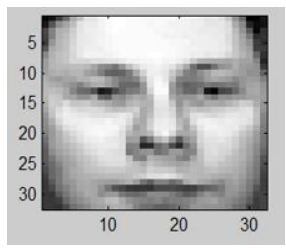

(b-1)

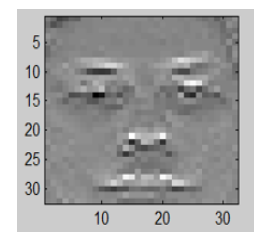

(b-2)

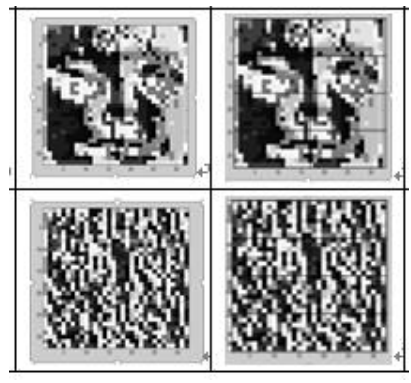

(b-3)

Figure 2. (a) First- and second-order decomposition based on wavelet transformation. (b) Examples of the decomposed first-order image of (a); in which (b-1) is an original image; (b-2) is a decomposed first-order image of (b-1); (b-3) is a decomposed second -order image of $L L_{1}$.

\section{Face-Recognition Model and SVM Scheme for User Authentication}

This section presents a face-recognition model with distributed facial features for an online e-commerce security system. The proposed model comprises two phases, namely the registration phase and verification phase.

\subsection{Basic Concept}

Numerous face-recognition techniques have been proposed to enhance identity recognition precision. To achieve high processing efficiency in an identity authentication system, numerous studies on face recognition have employed LBPs to obtain a low-resolution face image from captured facial features. The present study proposed a face-recognition technique based on a secret-splitting concept (Wang et al. 2011). In this concept, only some of the facial features of the user, extracted using twodimensional wavelet transformation and an LBP scheme, are stored in a smart card to avoid the risk of having the complete biometric data stored in a repository being stolen. Unlike existing schemes, the system provides the benefit of complete privacy protection through distributed storage, in which the smart card stores images of different facial expressions and a database contains records of face images.

This study proposes using a smart card and an SVM-based face-recognition model 
with distributed facial features in an online e-commerce security system (Fig. 3); the model involves wavelet transformation and provides multilevel analytical diagrams with information integrity. The wavelet transformation decomposes the face image into a face image with a low resolution, called a low-resolution image feature (LRIF), and a face image with a high resolution, called a high-resolution image feature (HRIF). The LRIF is stored in a smart card, and the HRIF is stored in a signature database for comparison. Notably, this study used both wavelet transformation and an LBP technique to extract regional (partial) facial features for enabling comparison in an online e-commerce security system. The wavelet transformation of a face image can be decomposed into two types of face images: (i) a series of LRIFs with different poses and (ii) a set of HRIFs corresponding to the LRIFs. An LBP technique is a simple and fast texture-handling approach; sheets of high-resolution characteristics of the face are transformed into feature histograms based on various spectral types.

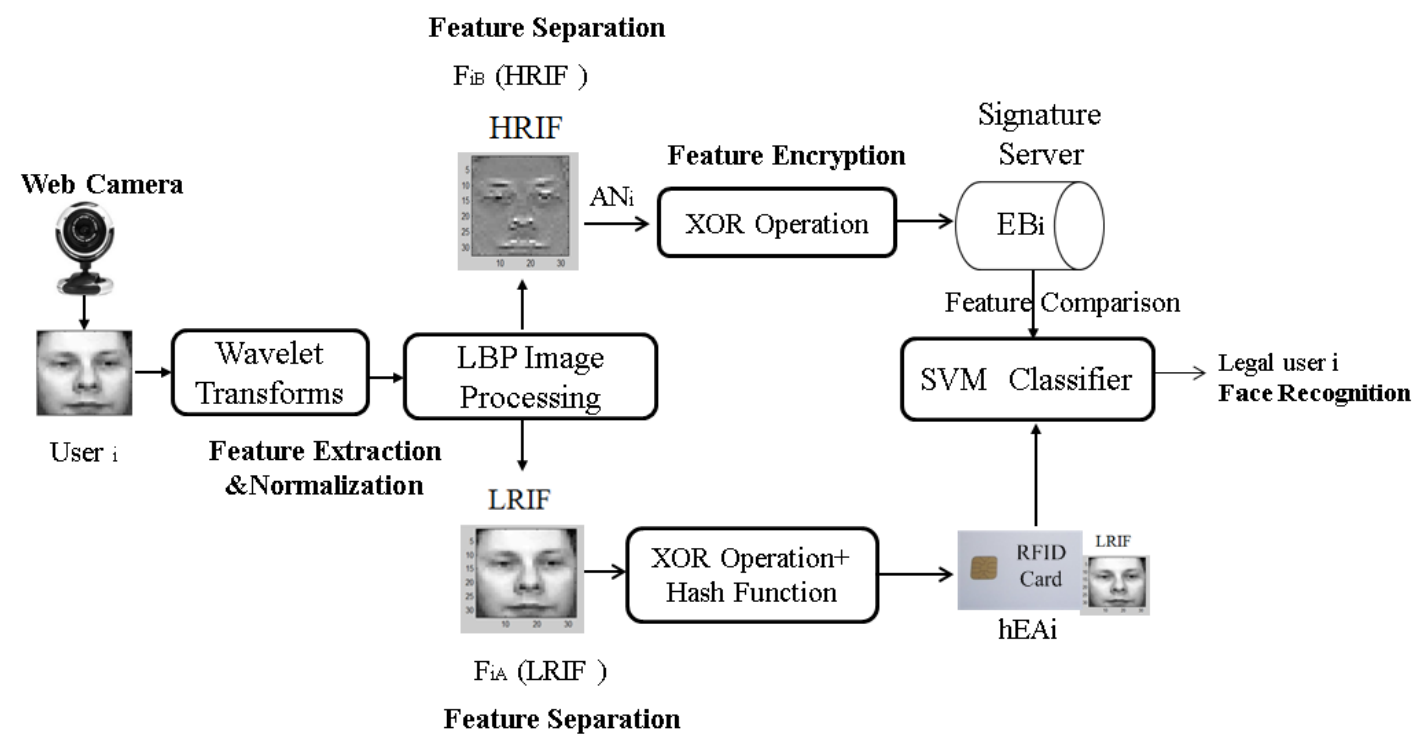

Figure 3. Basic concept of user authentication by using secret splitting.

\subsection{Proposed Face-Recognition Approach for User Authentication}

When a smart card manufacturer accepts an order from a service provider, the manufacturer incorporates various security parameters into the cards and then sends the cards to the service provider. The detailed procedure is shown in Fig. 4, which presents a function flow diagram of the proposed face-recognition scheme; the figure also illustrates the two subphases in the user authentication process: the registration phase and verification phase. 


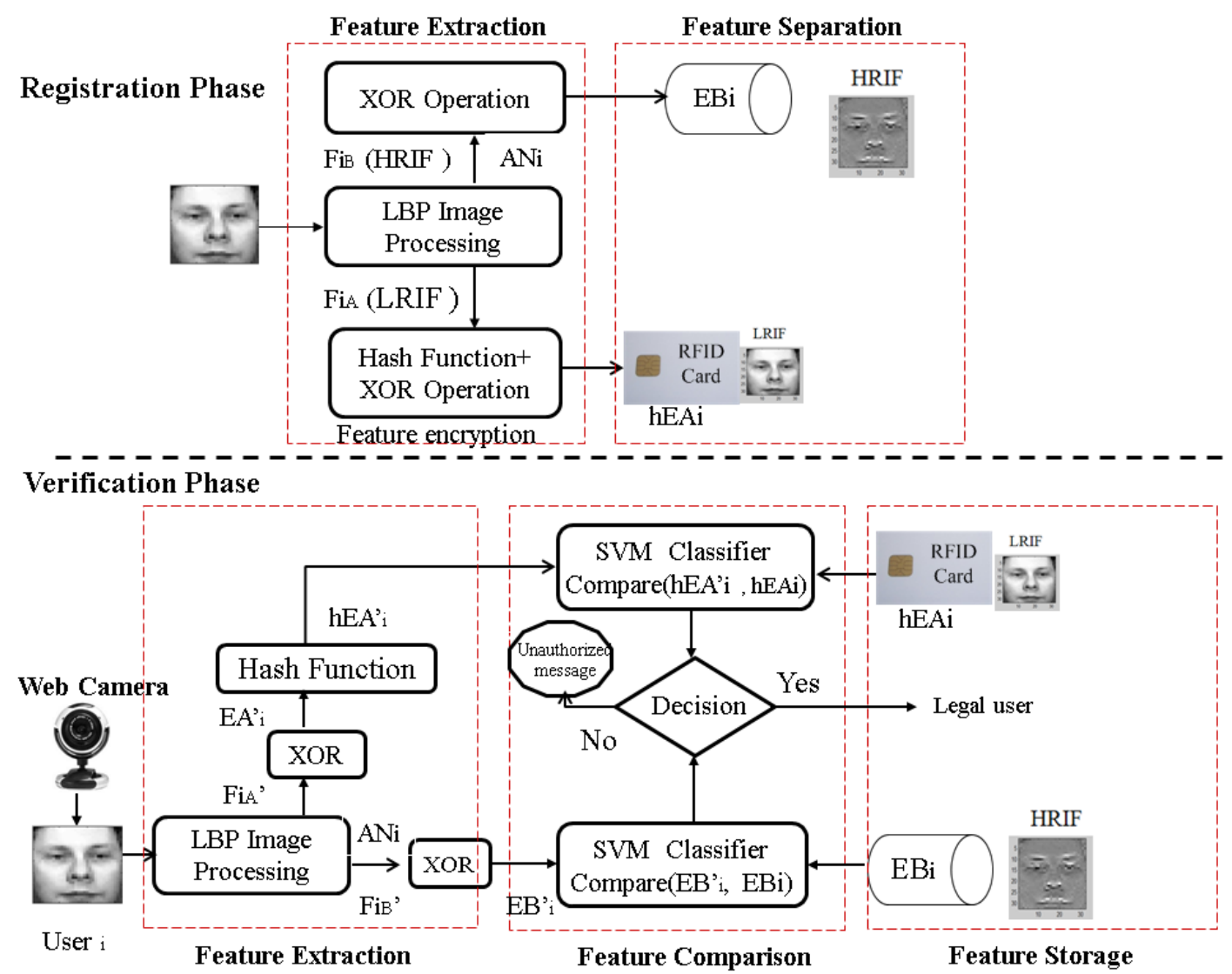

Figure 4. Proposed face-recognition scheme for user feature comparison.

\subsubsection{Registration Phase}

In the registration phase, various expressions in the face images of registered users are decomposed into LRIFs and HRIFs for face recognition in an access control system. The LRIFs are stored in a compressed format and encrypted in a smart card, and the HRIFs are stored in a signature database. As shown in Fig. 4, the registration phase comprises the following four steps:

1) User $U_{i}$ with identity $\operatorname{ID}_{i}$ wants to register with the server. The user chooses a smart card, and then LRIFs are protected by the encryption mechanism of the smart card.

2) A face image of User $U_{i}$ is obtained through a Web camera, and the features are extracted from this image to form a face feature $F_{i}$ which are separated into two parts $\mathrm{F}_{\mathrm{iA}}$ and $\mathrm{F}_{\mathrm{iB}}$, where $\mathrm{F}_{\mathrm{iA}}$ and $\mathrm{F}_{\mathrm{iB}}$ represent Part $\mathrm{A}$ (i.e., the LRIF) and Part B (i.e., the HRIF), respectively.

3) The terminal computes $h E A_{i}=h\left(F_{i A} \oplus A_{i}\right)$ and $\mathrm{EB}_{i}=\left(F_{i B} \oplus A_{i}\right)$, where $h($.$) is$ a public one-way hash function and $\oplus$ represents the exclusive-or operator (XOR).

4) The card manufacturer arbitrarily selects a large prime number $p$ and a 128-bit string $\mathrm{K}$ as the key for symmetric encryption and keeps the parameter set ( $\mathrm{p}, \mathrm{AN}$, $\mathrm{K})$ secret. The terminal sends $\left(\mathrm{ID}_{\mathrm{i}}, \mathrm{EB}_{\mathrm{i}}, \mathrm{p}, \mathrm{K}\right)$ to the server over a secure channel. The terminal then stores $\left(\mathrm{ID}_{\mathrm{i}}, \mathrm{hEA}_{\mathrm{i}}\right)$ in the smart card, and the signature server stores $\left(\mathrm{ID}_{\mathrm{i}}, \mathrm{EB}_{\mathrm{i}}\right)$ in the database of an access control system. 
In the verification phase, the LRIFs of registered users are retrieved from the encrypted smart card for the first-phase comparison. The HRIFs are then retrieved from the database for the second-phase comparison. Fig. 4 shows the details of the verification phase.

5) User $U_{i}$ places his or her RFID card on the terminal. If the RFID card is validated, AN is extracted; otherwise, the login request is rejected.

6) Users provide their images through a Web camera, and the facial features are then compared with those stored in both the smart card and signature server. Let Fi represent the facial features extracted by the Web camera. The image processing unit separates $F_{i}$ into $F_{i A}$ and $F_{i B}$ and then computes $h E A_{i}=h\left(F_{i A} \oplus A N\right)$ and $E_{i}=$ $\left(\mathrm{F}_{\mathrm{iB}} \oplus \mathrm{AN}\right)$; here, $\mathrm{h}$ represents a cryptographic hash function, such as a secure hash algorithm.

7) To verify the user's legal identity using the SVM algorithm, part A of facial feature, $\mathrm{hEA}_{\mathrm{i}}$ is retrieved for comparison. If a pose of facial image with a hyperplane that has the largest distance from the nearest training data point of any class, which is selected as one of candidates with SVM classifier in first verification phase.

8) Similarly, the server sends $E_{i}$ via a secure channel to the terminal for comparison purposes using the SVM algorithm. Use the selected candidates in step 7 to compare the alternatives for the maximum-margin hyperplane based on LBP feature histograms of HRIF for face image. If a match is obtained using the SVM classifier algorithm for HRIF image classification of $\mathrm{EB}_{\mathrm{i}}{ }^{\prime}$, the verification process proceeds to invoke an authorized application; else it terminates.

Because the SVC is an effective scheme for data classification, it is used to match the face features used for identifying the hyperplane that has the largest minimum distance from the training examples in Fig. 1.

In decision-making for face recognition, we tested the classification of a sample by using LBP feature histograms and HRIF matching; a decision function $D\left(x_{i}\right)$ was used to determine the SVC. The decision function was given by $D\left(x_{i}\right)=w \cdot x_{i}+b$. The training process was performed to select the hyperplane, and the classification system substituted the trained model parameters derived from the training data into the SVM to categorise the sample class for testing the data. In other words, the positive class $(+1)$ was predicted if $D\left(x_{i}\right)>0$, and the negative class (-1) was predicted otherwise.

\subsection{Assessing the global precision rate for the proposed scheme}

Two precision metrics, recognition precision for both the LRIF and the HRIF in Sec 3.2.2 are used for assessing the global precision rate is described as follows.

1) Determine the number of remaining alternatives in the facial feature database after performing the successful recognition for the LRIF as

$$
N_{\text {remaining }}=C_{\text {cardinalig }}\left(\left[u_{1}, \ldots, u_{n}\right]-\left[u_{1}, \ldots, u_{n}\right]_{L R I F}\right) \text {, }
$$

where $\left[u_{1}, \ldots, u_{n}\right]_{L R I F}$ and $\left[u_{1}, \ldots, u_{n}\right]_{H R I F}$ represents a set of successful recognition for 
the LRIF and the HRIF, respectively. $C_{\text {cardinaliy }}$ represents the cardinality of a set of images.. $N_{\text {remaining }}$ stands for the number of a set of remaining alternatives after performing the successful recognition for the LRIF. $\left[u_{1}, \ldots, u_{n}\right]$ denotes a full set of images stored in the facial feature database.

2) To increase the precision rate of face recognition, a set of the extra alternatives of successful recognition for the HRIF which are located in the remaining set $\left[u_{1}, \ldots, u_{n}\right]-\left[u_{1}, \ldots, u_{n}\right]_{L R I F}$ is decide by

$$
\left[u_{1}, \ldots, u_{n}\right]_{H_{R I F} \text { EXTRA }}=\left[u_{1}, \ldots, u_{n}\right]_{\text {HRIF }} \cap\left(\left[u_{1}, \ldots, u_{n}\right]-\left[u_{1}, \ldots, u_{n}\right]_{\text {LRIF }}\right)
$$

3) The global precision rate for the proposed scheme which synthesizes two precision rates for both the LRIF and the HRIF is given by

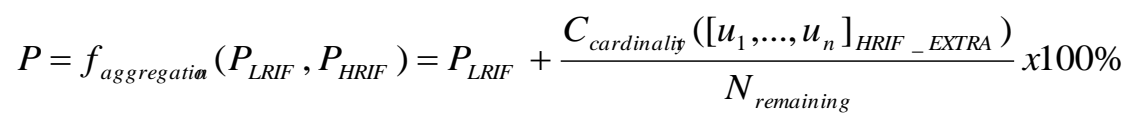

where $P_{\text {LRIF }}, P_{\text {HRIF }}$ represents the recognition precision for the LRIF and the HRIF, respectively. $f_{\text {aggregation }}$ denotes an aggregation function used in generating the global precision rate from $P_{L R I F}$, Eqs.(5) and (6).

\section{Experimental Results}

This section discusses the effectiveness of the proposed scheme for facial-featurebased user authentication in the e-commerce transactions. Consider a remote biometric-based authentication system for an open access network. The authentication system typically consists of a personal computer equipped with a Web camera and a smart card reader. This illustrative example focuses on information security in the context of an online transaction system. When users use a secure electronic transaction system, they regularly have to pass security authentication by specifying a secret authentication token in each request through a user terminal; the user terminal is equipped with a smart card reader and a Web camera that records the images of users and their usernames/passwords to generate a token for online authentication. In practice, most users use a personal smart card to receive authorisation to interact with services and ensure secure data access, and they store registration information on the smart card and the signature server in advance.

Initially, when a request is placed on the electronic transaction system to generate an authentication token for a user, the user must provide his or her personal information, including the LRIF and HRIF of his or her face image and his or her username/password to sign in and authorise the application to perform certain actions. When an unregistered user attempts to pass biometric authentication, the Web camera can capture face images of the user in real time and the authentication system can compare five images through the LRIF and HRIF matching processes (see Steps 7 and 8 , and Fig. 4) to verify the identity of the user. In other words, the system can automatically detect suspect users by retrieving LRIF and HRIF information for matching their face features.

An experiment was performed to validate the proposed face-recognition scheme could be provided, where user faces from both the Olivetti Research Laboratory (ORL) 
database (AT\&T Corp. 2014) and Face detection database of NCKU (NCKU 2014) were used for making comparisons and to calculate the recognition precision of the proposed scheme. The ORL contains five different images for each of 40 distinct subjects. The images of five of these subjects were used as learning samples, and the images of five of the other subjects were then used as the test samples for determining the face recognition precision. For some subjects, the lighting, facial expressions, and facial details varied in the five images. Each image had a size of $92 \times 112$ pixels, with 256 grey levels per pixel. Fig. 5 shows some sample images in the ORL database. The NCKU image database contains five different images of each of 10 distinct subjects. In this experiment, the subjects were divided into two groups with equal numbers of subjects. The images of one group were used as learning samples, and those of the other group were used as test samples.

In the experiment, feature extraction involved wavelet decomposition. A Haar wavelet (Charles 1992) was used to separate the features into two parts, and an LRIF was generated for each pose. A total of five pose images were stored in the encrypted smart card. In addition, six HRIFs were generated for every LRIF of a subject. The six HRIFs were then separated into two levels - first-level and second-level decomposition information-with each level consisting of three HRIF images (Fig. 2). Finally, six HRIF images were sent to a signature server for SVC-based face recognition.

Next, a test experiment was conducted for computing the distance between a point and a hyperplane by using an SVC to discriminate between neutral and various other facial expressions. Fig. 6 shows a face image of a subject with first-level decomposition; the subject was chosen from the test samples. Fig. 7 shows the LBP histograms corresponding to Fig. 6. Fig.7 indicates that each image was equally divided into eight parts for statistical analysis of partial facial features using LBP histograms in order to quantify differences among image samples in detail.

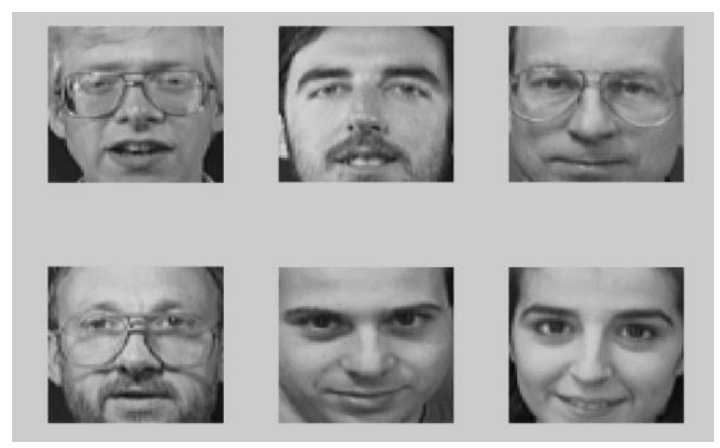

Figure 5. Sample images from the ORL database.

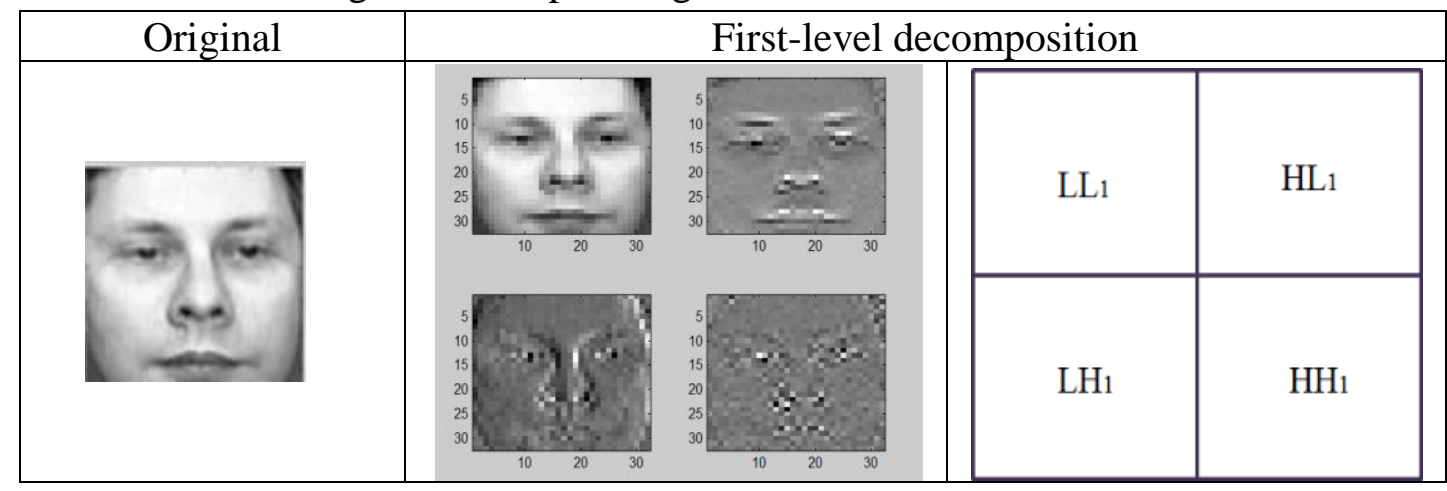

Figure 6. Face image with first-level decomposition. 

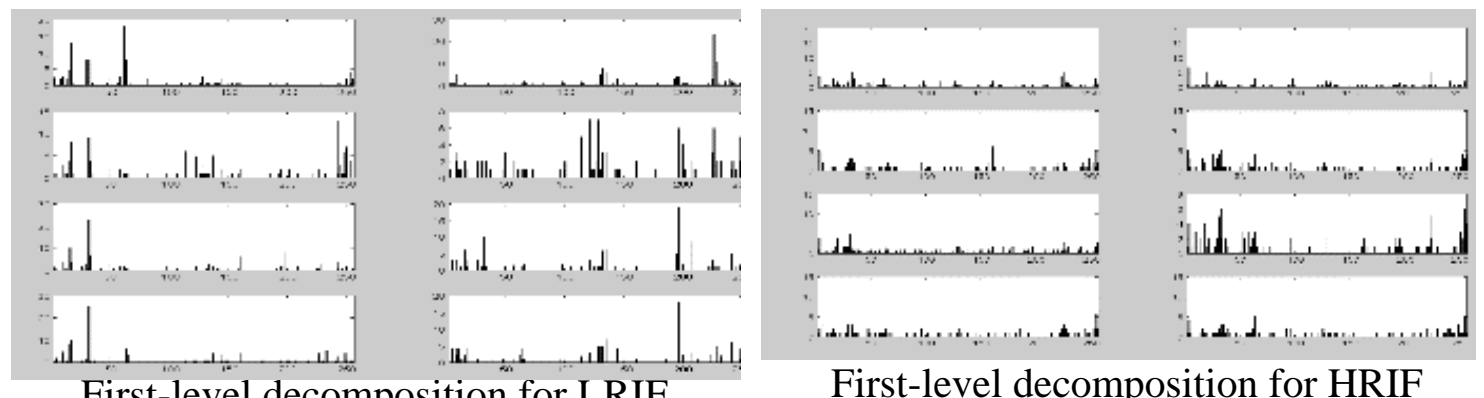

First-level decomposition for LRIF

First-level decomposition for HRIF

Figure 7. LBP histogram corresponding to Fig.6.

The ORL database contains ten different images of each of the 40 distinct subjects. To evaluate the proposed method efficiently, we filtered out photos with incomplete face information in the ORL database and obtained 341 complete face images for feature extraction. These images were normalised to a size of $64 \times 64$ pixels and were used in the following experiments. A frequently used SVM toolbox, LIBSVM (Hsu 2002, Lin and Lin 2003), was employed to implement the following analysis process.

1) Kernel function selection:

In the implementation of the aforementioned approach to determine the SVC, choosing a suitable kernel function theoretically for the LRIF and HRIF was difficult since the convergence value for the kernel parameters in face recognition varies with the image. Table 3 and Fig. 8 show that the kernel choice for the SVC affects the precision of face recognition. Thus, on the basis of the statistical characteristics of the test samples, the linear kernel or the RBF kernel was considered appropriate.

Table 3. Success percentage for the use of different kernel functions for SVM.

\begin{tabular}{|c|c|c|c|c|}
\hline $\begin{array}{c}\text { Sizing of } \\
\text { training } \\
\text { dataset }\end{array}$ & $\begin{array}{c}\text { Sizing of } \\
\text { testing } \\
\text { dataset }\end{array}$ & Kernel & $\begin{array}{c}\text { Number of success } \\
\text { recognition/ Number } \\
\text { of total images }\end{array}$ & $\begin{array}{c}\text { Recognition } \\
\text { Precision }\end{array}$ \\
\hline 117 & 224 & Linear & $195 / 224$ & $87.1 \%$ \\
\hline 117 & 224 & Polynomial & $182 / 224$ & $81.0 \%$ \\
\hline 117 & 224 & RBF & $191 / 224$ & $85.0 \%$ \\
\hline 156 & 185 & Linear & $165 / 185$ & $89.0 \%$ \\
\hline 156 & 185 & Polynomial & $155 / 185$ & $83.8 \%$ \\
\hline 156 & 185 & RBF & $158 / 185$ & $85.4 \%$ \\
\hline
\end{tabular}




\section{Recognition precision using three kernel functions for \\ SVM classifier}

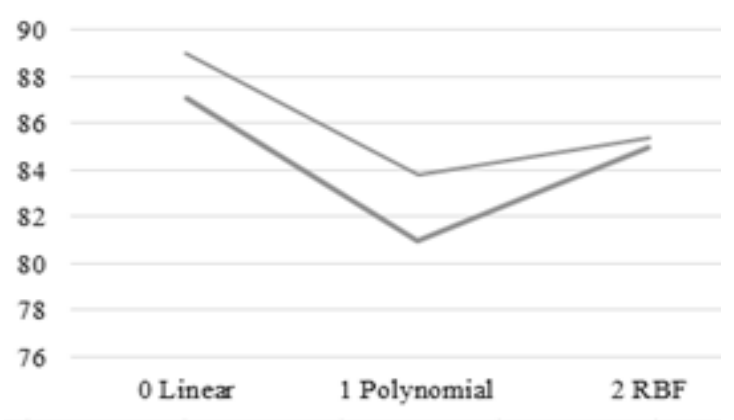

Figure 8. Recognition precision when three kernel functions were used

As shown in Fig. 4, there are two sub-phases of verification included in the facial recognition process: verification phase for low-resolution parts of facial features (phase I) and verification phase for high-resolution parts of facial features (phase II).

2) Precision verification for low-resolution parts of facial features (phase I):

A cross-validation scheme was adopted to predict identification precision for overcoming the overtraining problem by using various $n$-folds of the cross-validation scheme; for example, $k=10$ means that $90 \%$ of the dataset collected was used in the training experiment, and the remaining $10 \%$ of the dataset was used for alternative testing repeated 10 times. Since wavelet transform is a multiresolution waveletanalysis technique, we initially used a decomposed first-order face image $\mathrm{LL}_{1}$ and the cross-validation method $(k: 2-10)$ with the linear kernel function for the SVC; the results are shown in Table 4. The recognition precision increased with the number of training images for the LRIFs. Table 5 shows that the average success rate of facial recognition for the LRIF was approximately $95.3 \%$ higher than that of the scheme proposed in (Battaglia et al. 2014), where VisilabFaceRec uses a portion of the image information.

Table 4. Recognition precision for the LRIF for the linear kernel with the cost

\begin{tabular}{|c|c|}
\hline \multicolumn{2}{|c}{ parameter $(C=1)}$. \\
\hline n-fold & Recognition precision \\
\hline $\mathrm{k}=2$ & $90.90 \%$ \\
\hline $\mathrm{k}=3$ & $92.96 \%$ \\
\hline $\mathrm{k}=4$ & $95.89 \%$ \\
\hline $\mathrm{k}=5$ & $96.19 \%$ \\
\hline $\mathrm{k}=6$ & $95.89 \%$ \\
\hline $\mathrm{k}=7$ & $96.77 \%$ \\
\hline $\mathrm{k}=8$ & $95.89 \%$ \\
\hline $\mathrm{k}=9$ & $96.48 \%$ \\
\hline $\mathrm{k}=10$ & $96.77 \%$ \\
\hline Average precision & $95.30 \%$ \\
\hline
\end{tabular}


*Note: $\mathrm{C}=\left|2^{9}, \quad 2^{10}, \quad \ldots, \quad 2^{20}\right|$

3) Precision verification for high-resolution parts of facial features (phase II):

Once decided the recognition precision for the LRIF, we then calculate the recognition precision for the HRIF which is used for reconfirming the solution for the LRIF. Spatially enhanced LBP histograms (Mirza et al. 2013) for facial features were used to increase the recognition precision. An image was divided into four nonoverlapping blocks, $\mathrm{LL}_{1}, \mathrm{LH}_{1}, \mathrm{HL}_{1}$, and $\mathrm{HH}_{1}$ (Fig. 2). The LBP histogram was calculated for each block, and it was necessary to concatenate four histograms. The experimental results for the linear kernel with the cost parameter $(C=0.25)$ are shown in Table 5. It is clear that a high precision percentage was achieved when $\mathrm{HL}_{1}+\mathrm{LH}_{1}+\mathrm{HH}_{1}$ was used compared to that of $\mathrm{HL}_{1}$. Obviously, the computational load for $\mathrm{HL}_{1}$ was lower than that of $\mathrm{HL}_{1}+\mathrm{LH}_{1}+\mathrm{HH}_{1}$, and it can be reduced by including additional computation for concatenating three values of histograms $\left(\mathrm{HL}_{1}, \mathrm{LH}_{1}\right.$, and $\mathrm{HH}_{1}$ ). For simplicity, $\mathrm{HL}_{1}$ can be considered to represent the high-resolution part of face features (i.e., the HRIF) in face recognition.

Table 5. Recognition precision when spatially enhanced LBP histograms were used.

\begin{tabular}{|c|c|c|c|c|c|}
\hline $\begin{array}{c}\text { Sizing of } \\
\text { training } \\
\text { images }\end{array}$ & $\begin{array}{c}\text { Sizing of } \\
\text { testing } \\
\text { images }\end{array}$ & $\begin{array}{c}\text { Recognition } \\
\text { precision in } \\
\left.\text { phase I ( } \mathrm{LL}_{1}\right)\end{array}$ & $\begin{array}{c}\text { Recognition } \\
\text { precision in } \\
\text { phase II }\left(\mathrm{HL}_{1}\right)\end{array}$ & $\begin{array}{c}\text { Recognition } \\
\text { precision in phase } \\
\text { II } \\
\left(\mathrm{HL}_{1}+\mathrm{LH}_{1}+\mathrm{HH}_{1}\right)\end{array}$ & $\begin{array}{c}\text { The global } \\
\text { Recognition } \\
\text { precision } \\
\left(\mathrm{LL}_{1},\right. \\
\left.\mathrm{HL}_{1}+\mathrm{LH}_{1}+\mathrm{HH}_{1}\right)\end{array}$ \\
\hline 40 & 301 & $70.2 \%$ & $57.9 \%$ & $56.6 \%$ & $74.30 \%$ \\
\hline 80 & 261 & $82.1 \%$ & $74.5 \%$ & $74.9 \%$ & $83.25 \%$ \\
\hline 120 & 221 & $87.1 \%$ & $77.2 \%$ & $79.0 \%$ & $88.90 \%$ \\
\hline 160 & 181 & $89.0 \%$ & $84.3 \%$ & $84.9 \%$ & $92.25 \%$ \\
\hline 195 & 146 & $95.9 \%$ & $88.4 \%$ & $86.3 \%$ & $97.30 \%$ \\
\hline 233 & 108 & $97.8 \%$ & $86.1 \%$ & $87.0 \%$ & $98.95 \%$ \\
\hline 267 & 74 & $97.3 \%$ & $90.5 \%$ & $87.8 \%$ & $98.95 \%$ \\
\hline 296 & 45 & $97.7 \%$ & $88.9 \%$ & $91.1 \%$ & $98.95 \%$ \\
\hline
\end{tabular}

From Table 5, it is evidently that the highest precision rate is gain in $\mathrm{LL}_{1}$ compared to that of the high-resolution part of face features due to the fact that most facial features were covered in the LRIF.

Similarly, experiments in which $n$ folds of the cross-validation scheme were used were conducted for the HRIF. Table 6 shows that the corresponding recognition precision for the HRIF was approximately $87.62 \%$ when the cross-validation method was used (k:210) along with the linear kernel function for the SVM.

Table 6. Recognition precision when the linear kernel with the cost parameter $(C=1$.) for the HRIF.

\begin{tabular}{|c|c|}
\hline n-fold & Recognition precision \\
\hline $\mathrm{k}=2$ & $81.82 \%$ \\
\hline $\mathrm{k}=3$ & $86.80 \%$ \\
\hline
\end{tabular}




\begin{tabular}{|c|c|}
\hline $\mathrm{k}=4$ & $86.80 \%$ \\
\hline $\mathrm{k}=5$ & $88.27 \%$ \\
\hline $\mathrm{k}=6$ & $88.27 \%$ \\
\hline $\mathrm{k}=7$ & $90.02 \%$ \\
\hline $\mathrm{k}=8$ & $88.56 \%$ \\
\hline $\mathrm{k}=9$ & $89.15 \%$ \\
\hline $\mathrm{k}=10$ & $88.86 \%$ \\
\hline $\begin{array}{c}\text { Average } \\
\text { precision }\end{array}$ & $87.62 \%$ \\
\hline
\end{tabular}

*Note: $\mathrm{C}=\left|2^{9}, \quad 2^{10}, \quad \ldots, \quad 2^{20}\right|$

4) The global precision rate of face recognition

By using Eqs.(5) (7), the global precision rate of face recognition is determined by the synthetic evaluations of recognition results of both the LRIF (i.e., $\mathrm{LL}_{1}$ ) and the HRIF(i.e., $\mathrm{HL}_{1}+\mathrm{LH}_{1}+\mathrm{HH}_{1}$ ), as shown in the last column of Table 5. Obviously, the global precision rate of proposed scheme provides a higher precision of face recognition compared with that of for the LRIF and the HRIF.

5) Robustness of the proposed method:

To demonstrate the robustness of the proposed method, we conduct experiments with a large facial image database, FERET (Phillips et al. 1998, Phillips et al. 2000). The selected images that include 24 individuals with seven different expressions, which are neutral, happiness, sadness, surprise, fear, anger, and disgust, as shown in Fig. 9. These images were normalised to a size of $256 \times 256$ pixels, with 256 grey levels and were used in the following experiments.
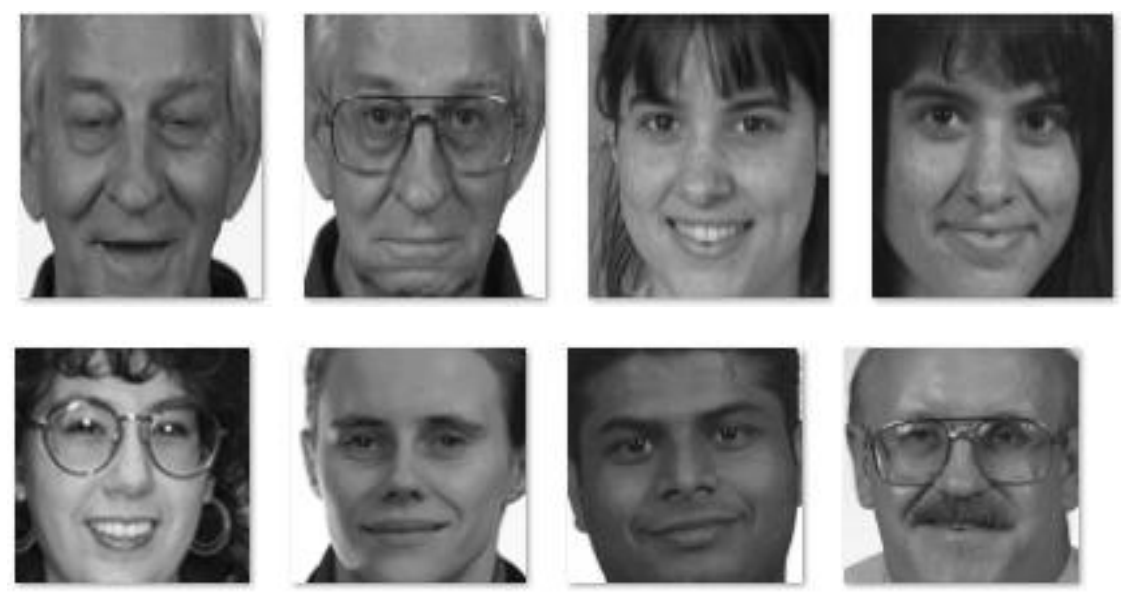

Figure 9. Sample images from the FERET database.

In the experiment, wavelet transform to decompose fourth-order for each face image, strengthen the extraction of space features with spatially enhanced LBP in the precision prediction with cross-validation scheme. By using wavelet transformation technique, the face image is subjected to an $\mathrm{LL}_{4}, \mathrm{LH}_{4}, \mathrm{HL}_{4}$, or $\mathrm{HH}_{4}$ band of decomposition with fourth-order decomposition. The experiments in which $n$ folds of the cross-validation scheme were used for the HRIF and the experimental results for the RBF kernel were 
shown in Table 7. Table 7 shows that the corresponding recognition precision for the HRIF was approximately $96.18 \%$. The experimental results for the RBF kernel with the cost parameter $(C=4)$ are shown in Table 8 . Table 8 shows that it can increase the recognition precision for the HRIF when spatially enhanced LBP histograms were used.

Finally, the global precision rate is determined by the synthetic evaluations of recognition results of both the LRIF and the HRIF, as shown in Table 9. The global precision rate for various combinations of components (low-resolution and highresolution face image from captured facial features) for both the LRIF and the HRIF is shown in Fig.10. Fig.10 shows that spatially enhanced LBP histograms for facial features can increase the recognition precision.

Overall, the proposed scheme provided a higher precision of face recognition compared with the average precision for face recognition (approximately 89\%) of the schemes proposed in (Ojala et al. 1996, Jing et al. 2009, Tan and Triggs 2010, Nguyen et al. 2012, Yang et al. 2012, Battaglia et al. 2014).

Table 7. Recognition precision when the RBF kernel for the HRIF.

\begin{tabular}{|c|c|c|c|}
\hline $\mathrm{n}$-fold & $\begin{array}{c}\text { Recognition } \\
\text { Precision } \\
(\%)\end{array}$ & $C($ cost $)$ & $\gamma($ gamma $)$ \\
\hline $\mathrm{k}=2$ & $90.63 \%$ & 32 & $3.05175781250000 \mathrm{e}-05$ \\
\hline $\mathrm{k}=3$ & $95.63 \%$ & 32 & $1.52587890625000 \mathrm{e}-05$ \\
\hline $\mathrm{k}=4$ & $95.00 \%$ & 32 & $1.52587890625000 \mathrm{e}-05$ \\
\hline $\mathrm{k}=5$ & $95.63 \%$ & 8 & $6.10351562500000 \mathrm{e}-05$ \\
\hline $\mathrm{k}=6$ & $96.88 \%$ & 4 & 0.000122070312500000 \\
\hline $\mathrm{k}=7$ & $98.13 \%$ & 16 & $3.05175781250000 \mathrm{e}-05$ \\
\hline $\mathrm{k}=8$ & $98.75 \%$ & 16 & $3.05175781250000 \mathrm{e}-05$ \\
\hline $\mathrm{k}=9$ & $97.50 \%$ & 4 & 0.000122070312500000 \\
\hline $\mathrm{k}=10$ & $97.50 \%$ & 4 & 0.000122070312500000 \\
\hline $\begin{array}{c}\text { Average } \\
\text { precision }\end{array}$ & $96.18 \%$ & \multicolumn{3}{|l}{} \\
\hline
\end{tabular}

Table 8. Recognition precision for the HRIF when spatially enhanced LBP histograms were used. $(C=4)$

\begin{tabular}{|c|c|c|c|c|}
\hline $\begin{array}{c}\text { Sizing of } \\
\text { training } \\
\text { images }\end{array}$ & $\begin{array}{c}\text { Sizing of } \\
\text { testing } \\
\text { images }\end{array}$ & $\begin{array}{c}\text { Recognition } \\
\text { precision } \\
\left(\mathrm{LL}_{4}\right)\end{array}$ & $\begin{array}{c}\text { Recognition } \\
\text { precision } \\
\left(\mathrm{HL}_{4}\right)\end{array}$ & $\begin{array}{c}\text { Recognition } \\
\text { precision } \\
\left(\mathrm{HL}_{4}+\mathrm{HL}_{3}+\mathrm{HL}_{2}\right)\end{array}$ \\
\hline 24 & 136 & $78.68 \%$ & $62.50 \%$ & $72.79 \%$ \\
\hline 48 & 112 & $92.86 \%$ & $81.25 \%$ & $88.39 \%$ \\
\hline 72 & 88 & $95.45 \%$ & $80.68 \%$ & $86.36 \%$ \\
\hline 96 & 64 & $98.44 \%$ & $81.25 \%$ & $89.06 \%$ \\
\hline 120 & 40 & $97.50 \%$ & $75.00 \%$ & $85.00 \%$ \\
\hline 144 & 16 & $100.0 \%$ & $93.75 \%$ & $87.50 \%$ \\
\hline
\end{tabular}


Table 9. The global precision of face recognition

\begin{tabular}{|c|c|c|c|c|}
\hline $\mathrm{n}$-fold & $\begin{array}{c}\text { Recognition } \\
\text { precision } \\
\left(\mathrm{LL}_{4}\right)\end{array}$ & $\begin{array}{c}\text { Recognition } \\
\text { precision } \\
\left(\mathrm{HL}_{4}\right)\end{array}$ & $\begin{array}{c}\text { Recognition } \\
\text { precision } \\
\left(\mathrm{HL}_{4}+\mathrm{HL}_{3}\right. \\
\left.+\mathrm{HL}_{2}\right)\end{array}$ & $\begin{array}{c}\text { The global precision } \\
\left(\mathrm{LL}_{4}, \mathrm{HL}_{4}+\mathrm{HL}_{3}+\mathrm{HL}_{2}\right)\end{array}$ \\
\hline $\mathrm{k}=2$ & $91.88 \%$ & $85.63 \%$ & 90.63 & $94.82 \%$ \\
\hline $\mathrm{k}=3$ & $98.13 \%$ & $89.34 \%$ & 95.63 & $94.63 \%$ \\
\hline $\mathrm{k}=4$ & $98.13 \%$ & $88.13 \%$ & 95.00 & $94.80 \%$ \\
\hline $\mathrm{k}=5$ & $98.75 \%$ & $90.00 \%$ & 95.63 & $96.62 \%$ \\
\hline $\mathrm{k}=6$ & $99.38 \%$ & $90.00 \%$ & 96.88 & $97.58 \%$ \\
\hline $\mathrm{k}=7$ & $99.38 \%$ & $90.63 \%$ & 98.13 & $97.94 \%$ \\
\hline $\mathrm{k}=8$ & $99.38 \%$ & $90.63 \%$ & 98.75 & $98.52 \%$ \\
\hline $\mathrm{k}=9$ & $99.38 \%$ & $91.25 \%$ & 97.50 & $99.76 \%$ \\
\hline $\mathrm{k}=10$ & $99.38 \%$ & $91.25 \%$ & 97.50 & $100.00 \%$ \\
\hline $\begin{array}{c}\text { Average } \\
\text { precision }\end{array}$ & $98.20 \%$ & $89.65 \%$ & $96.18 \%$ & $97.19 \%$ \\
\hline
\end{tabular}

The global precision rate

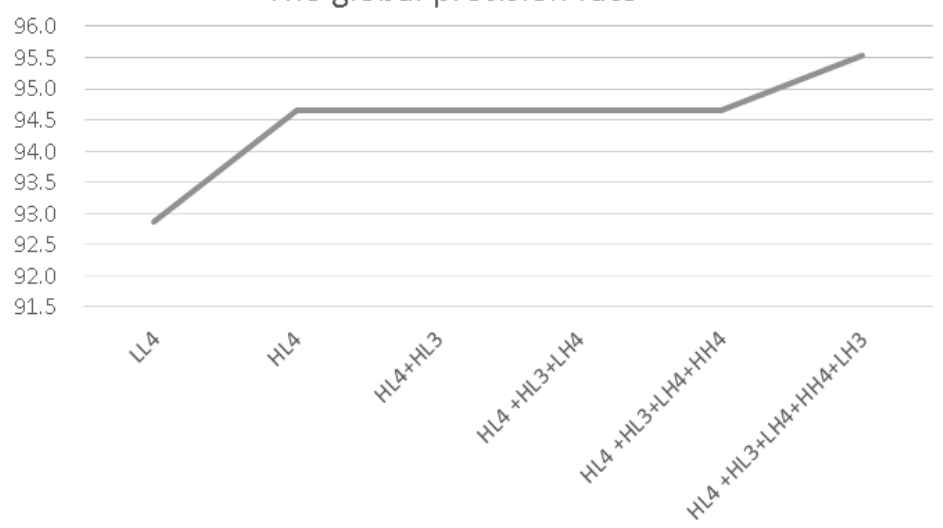

Figure 10. The global precision rate for various combinations of components for the LRIF and the HRIF.

\section{Discussion}

Choosing multiple parameters for SVMs is a major problem when multiple local optima exist. This section discusses the precision of SVM parameter selection in the face authentication process of the proposed scheme. In practice, it is necessary to ensure face recognition precision by considering the use of other kernel functions. Table 10 shows that selecting different kernels for the SVC affects the precision in a face-recognition experiment. Thus, the RBF kernel was used as an alternative kernel for the SVC for conducting training experiments based on statistical characteristics of test samples.

As described previously, 341 facial features were extracted from the ORL database in the training experiment. Once the SVMs were trained, they were used to authenticate users based on facial features. SVM parameters, including $C$ and $\gamma$, were derived from the facial features used for training (Altman, 1992, Goldberg and Elhadad 2008). The precision (\%) associated with the optimal $C$ and $\gamma$ values for the LRIF and 
HRIF using LIBSVM is shown in Table 7; the precision was determined using the cross-validation method $(\mathrm{k}: 2-10)$. The table shows that the prediction precision achieved using the RBF kernel function is close to that obtained using the linear kernel function. For the low-resolution and high-resolution face image samples, the face recognition precision was approximately $97.07 \%$ and $88.86 \%$ with tenfold crossvalidation, respectively.

Table 10. Face-recognition precision for the RBF kernel for different $n$-folds of the cross-validation scheme

\begin{tabular}{|l|c|c|c|c|c|c|}
\hline \multirow{2}{*}{ n-fold } & \multicolumn{3}{|c|}{ Face recognition for LRIF } & \multicolumn{2}{c|}{ Face recognition for HRIF } \\
\cline { 2 - 7 } & $\begin{array}{c}\text { Precision } \\
(\%)\end{array}$ & $C$ & $\gamma$ & $\begin{array}{c}\text { Precision } \\
(\%)\end{array}$ & $C$ & $\gamma$ \\
\hline $\mathrm{k}=2$ & 90.91 & 8 & $6.10 \mathrm{E}-05$ & 82.40 & 8 & $1.22 \mathrm{E}-04$ \\
\hline $\mathrm{k}=3$ & 93.26 & 32 & $1.53 \mathrm{E}-05$ & 86.51 & 16 & $6.10 \mathrm{E}-05$ \\
\hline $\mathrm{k}=4$ & 95.89 & 32 & $1.53 \mathrm{E}-05$ & 86.80 & 256 & $3.81 \mathrm{E}-06$ \\
\hline $\mathrm{k}=5$ & 96.48 & 2 & $2.44 \mathrm{E}-04$ & 88.27 & 256 & $3.81 \mathrm{E}-06$ \\
\hline $\mathrm{k}=6$ & 96.19 & 2 & $2.44 \mathrm{E}-04$ & 88.27 & 8 & $1.22 \mathrm{E}-04$ \\
\hline $\mathrm{k}=7$ & 96.77 & 8 & $6.10 \mathrm{E}-05$ & 90.03 & 16 & $6.10 \mathrm{E}-05$ \\
\hline $\mathrm{k}=8$ & 96.19 & 4 & $1.22 \mathrm{E}-04$ & 88.56 & 16 & $6.10 \mathrm{E}-05$ \\
\hline $\mathrm{k}=9$ & 96.77 & 8 & $6.10 \mathrm{E}-05$ & 89.15 & 64 & $1.53 \mathrm{E}-05$ \\
\hline $\mathrm{k}=10$ & 97.07 & 4 & $1.22 \mathrm{E}-04$ & 88.86 & 8 & $1.22 \mathrm{E}-04$ \\
\hline $\begin{array}{l}\text { Average } \\
\text { precision }\end{array}$ & 95.50 & & & 87.65 & & \\
\hline
\end{tabular}

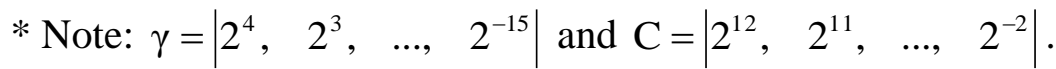

\section{Conclusions}

This paper presents a face-recognition SVM model based on a smartcard-based secret-splitting concept for preserving user privacy; Compared to existing schemes, the system has a number of important advantages, namely (i) provides the benefit of complete privacy protection through distributed storage, in which the smart card stores images of different facial expressions and a database contains records of face images; (ii) the model uses multilayered analytical diagrams for wavelet conversion and a dual pattern of a spatially enhanced LBP feature-histogram spectrum, and it improves the face recognition precision. An SVM algorithm was used for cross-validation scheme verification and favourable experimental results were obtained. Overall, the proposed approach can enhance the recognition precision compared with approaches proposed in (Ojala et al. 1996, Jing et al. 2009, Tan and Triggs 2010, Nguyen et al. 2012, Yang et al. 2012, Battaglia et al. 2014), reduce the possibility of disclosure of private user information in the registration and authentication phases, and protect the users' information against the theft of the user's facial features by interior dishonest staff by using secret-splitting mechanism. 


\section{Acknowledgment}

This work was supported jointly by the Ministry of Science and Technology of Taiwan under Grant Nos. MOST 104-2410-H-168-001 and MOST 103-2627-E-168001. Portions of the research in this paper use the FERET database of facial images collected under the FERET program, sponsored by the DOD Counterdrug Technology Development Program Office.

\section{References}

1. Abe S., 2015. Fuzzy support vector machines for multilabel classification. Pattern Recognition 48, 2110-2117.

2. Ahonen T., Hadid A., Pietikainen M., 2006. Face description with local binary patterns: application to face recognition. IEEE Transaction on Pattern Analysis and Machine Intelligence 28, 2037-2041.

3. Altman N.S., 1992. An introduction to kernel and nearest-neighbor nonparametric regression. The American Statistician 46 (3), 175-185.

4. An W., Liang M., 2013. Fuzzy support vector machine based on within-class scatter for classification problems with outliers or noises. Neurocomputing 110, 101-110.

5. AT\&T Corp., 2015. The Database of Faces. Retrieved from <http://www.cl.cam.ac.uk/research/dtg/attarchive/facedatabase.html> (accessed 2. January 2014)

6. Battaglia F., Iannizzotto G., Lo Bello L., 2014. A biometric authentication system based on face recognition and RFID tags. Mondo Digitale 13(49), 340-346.

7. Bernhard E.B., Isabelle M.G., Vapnik V., Vladimir N., 1992. A training algorithm for optimal margin classifiers. 5th Annual ACM Workshop on COLT, Pittsburgh, 144-152.

8. Buhmann M.D., 2003. Radial Basis Functions: Theory and Implementations. Cambridge University Press.

9. Charles K. C., 1992. Wavelets : A Tutorial in Theory and Applications. Academic Press, San Diego, USA.

10. Devi H.S., Laishram R., Thounaojam D.M., 2015. Face recognition using R-KDA with non-Linear SVM for multi-view Database. Procedia Computer Science 54, $532-541$.

11. Goldberg Y., Elhadad M., 2008. splitSVM: Fast, space-efficient, non-heuristic, polynomial kernel computation for NLP applications. In Proceedings of the 46st Annual Meeting of the Association of Computational Linguistics (ACL-08), 237240.

12. Gunn S., 1998. Support vector machines for classification and regression. ISIS 
Technical Report.

13. Hsu C.W., 2002. A comparison of methods for multiclass support vector machines. IEEE Transactions on Neural Networks 13(2), 415-425.

14. IT Security, 2015. The 10 Worst Security Incidents of 2014 Breaches (plus one). Retrieved from <http://www.it-security-inc.com/home/blog/security-hackingstories/the-10-worst-security-incidents-of-2014-breaches-plus-one> (accessed 9. February 2015).

15. Jing B.Z., Yeung D.S., Ng W.W.Y, Ding H.L., Wu D.L., Wang Q.C., Li J.C., 2009. RFID Access authorization by face recognition. 8th International Conference on Machine Learning and Cybernetics, Baoding, 302-307.

16. Liao W.H., 2010. Region description using extended local ternary patterns. International Conference on Pattern Recognition, 1003-1006.

17. Lin H.T., Lin C.J., 2003. A study on sigmoid kernels for SVM and the training of non-PSD kernels by SMO-type methods, Technical report. Department of Computer Science \& Information Engineering. National Taiwan University.

18. Min D.G., Kim J.W., Jun J.S., 2011. The entrance authentication system in realtime using face extraction and the RFID. International Conference on Ubiquitous Computing and Multimedia Applications (UCMA), 20-24.

19. Mirza A. M., Hussain M., Almuzaini H., Muhammad G., Aboalsamh H., Bebis G., 2013. Gender recognition using fusion of local and global facial features. Lecture Notes in Computer Science (LNCS) 8034, 493-502.

20. NCKU, Database for Face Detection. Retrieved from <http://robotics.csie.ncku.edu.tw/Databases/FaceDetect_PoseEstimate.htm> (accessed 20. April 2014)

21. Nguyen T.D., Quang L.D., Van N.C., Thanh L.T., Hoang T.M., De Souza-Daw T., 2012. An efficient and reliable human resource management system based on a hybrid of face authentication and RFID technology. 4th International Conference on Communications and Electronics (ICCE), 333-338.

22. Ojala, T., Pietikainen M., Harwood D., 1996. A comparative study of texture measures with classification based on feature distributions. Pattern Recognition 29, 51-59.

23. Phillips P.J., Wechsler H., Huang J., Rauss P., 1998. The FERET database and evaluation procedure for face recognition algorithms. Journal of Image and Vision Computing 16(5), 295-306.

24. Phillips, P.J. Moon H., Rizvi S.A., Rauss P.J., 2000. The FERET Evaluation methodology for face recognition algorithms. IEEE Trans. Pattern Analysis and Machine Intelligence 22, 1090-1104.

25. Tan X., Triggs B., 2010. Enhanced local texture feature sets for face recognition 
under difficult lighting conditions. IEEE Transaction on Image Processing 19(6), 1635-1650.

26. Vapnik V., 1995. The Nature of Statistical Learning Theory. Springer, New York.

27. Wang P., Ku1 C. C., Wang T. C., 2011. A new fingerprint authentication scheme based on secret-splitting for enhanced cloud security. Recent Application in Biometrics, 183-196.

28. Wikipedia, 2015, Support Vector Machine. Retrieved from <http://en.wikipedia.org/wiki/ Support_vector_machine> (accessed 14. March 2015).

29. Yang D., Xu B., Yang B., Wang J., 2012. A novel biometric authentication scheme with privacy preserving. 8th International Conference on Computational Intelligence and Security, 452- 456. 